\title{
Septic Pulmonary Emboli and Right Ventricular Obstruction in a Membranous Interventricular Septal Aneurysm
}

\author{
Mehdi Bamous, Abdessamad Abdou, Noureddine Atmani, Anis Sergouchni, \\ Younes Moutakiallah, Hatim Ghadbane, Mohamed Drissi, Abdelatif Boulahya \\ Department of Cardiovascular Surgery, Mohammed 5th Military Hospital, University Mohamed 5, Rabat, Morocco \\ Email: mehdibamous@hotmail.com
}

Received 28 May 2014; revised 28 June 2014; accepted 28 July 2014

Copyright (C) 2014 by authors and Scientific Research Publishing Inc. This work is licensed under the Creative Commons Attribution International License (CC BY). http://creativecommons.org/licenses/by/4.0/

\section{(c) (7) Open Access}

\begin{abstract}
Although the majority of patients with perimembranous ventricular septal defect and septal aneurysm remained asymptomatic, some of them presented with serious complications during adulthood and thus required high risky surgery. In accordance with other rare condition, the incidence and natural history have not been well documented. This case describes the occurrence of a septic pulmonary emboli associated with right ventricular outflow tract obstruction in a young child.
\end{abstract}

\section{Keywords}

Infective Endocarditis, Ventricular Septal Defect, Membranous Septum Aneurysm, Right Ventricular Obstruction

\section{Introduction}

Aneurysm of membranous septum (AMS) develops as a consequence of spontaneous closure of perimembranous ventricular septal defect (VSD).

Clinical presentation ranges from asymptomatic patients to complications such as endocarditis, thrombosis, rupture and right ventricular outflow tract obstruction (RVOTO).

The diagnosis in asymptomatic patients is usually made accidentally by transthoracic echocardiography.

We describe the occurrence of septic pulmonary embolism and right ventricular outflow tract obstruction in a 
young child.

\section{Case Report}

A previously healthy 8-year-old girl presented to our hospital with fever and shortness of breath. On admission, her blood pressure was 100/65 mmhg, a heart rate of 90 cycles/min, a respiratory rate of 25 breaths/min and a fever of $38.5^{\circ}$. On Cardiopulmonary exam, a systolic ejection murmur in the left upper sternal border was detected associated with a decreased breath sound in the right lower lung field. The electrocardiogram showed sinus rhythm with an incomplete right bundle branch block. Chest x-ray revealed a cardiomegaly with parenchymal opacities in the right lower lobe. Transthoracic echocardiography (TTE) showed a restrictive perimembranous VSD (Figure 1(a)) and a giant aneurysm of the membranous septum protruding into the right ventricle and obstructing the pulmonary trunk, there is a small vegetation attached to the AMS (Figure 1(b)). A jet velocity of $5 \mathrm{~m} / \mathrm{s}$ across RVOT was measured by color doppler associated with right ventricular hypertrophy. The inflammatory parameters were elevated and blood cultures were positive for streptococcus viridans. The diagnosis of infective endocarditis was made and the patient was treated accordingly with intravenous penicillin (4 weeks) and gentamicin (2 weeks). Additionally a computed tomography angiography (CT) confirmed the AMS protruding into the pulmonary trunk (Figure 2(a)) and showed directly the emboli obstructing a segmental artery of the right inferior lobe associated with a wedge-shaped, triangular image due to pulmonary infarction (Figure 2(b)), cerebral and abdominal CT were normal. The patient was immediately referred for surgery; on inspection the right atrium and ventricle were moderately hypertrophied; the AMS was exposed via a right atrium and pulmonary artery; it was globally inflamed with some granulations without signs of thrombus formation. The aneurysm was excised completely and the VSD was closed with an appropriately sized Dacron patch. The recovery was uneventful and the child was discharged from the hospital four weeks after admission. The bacterial culture of the aneurysm was sterile.

\section{Discussion}

Morphologically, the aneurysm tissue derives from the septal leaflet of tricuspid valve and the membranous part of the interventricular septum in the presence of VSD [1]. Aneurysm formation functionally reduces the left to right shunt but had the potential consequence of promoting RVOTO, bacterial endocarditis, rupture and thromboembolism [2].

TTE is an effective tool for the diagnosis of AMS; however its morphological evaluation lacks detail. In the setting of perimembranous aneurysm, the sensitivity of TTE is 70\% [3]. Cardiac magnetic resonance imaging and CT provide additional information regarding the structure and the extent of the aneurysm.

Our patient had a markedly right ventricular hypertrophy attesting of a chronic RVOTO associated with a

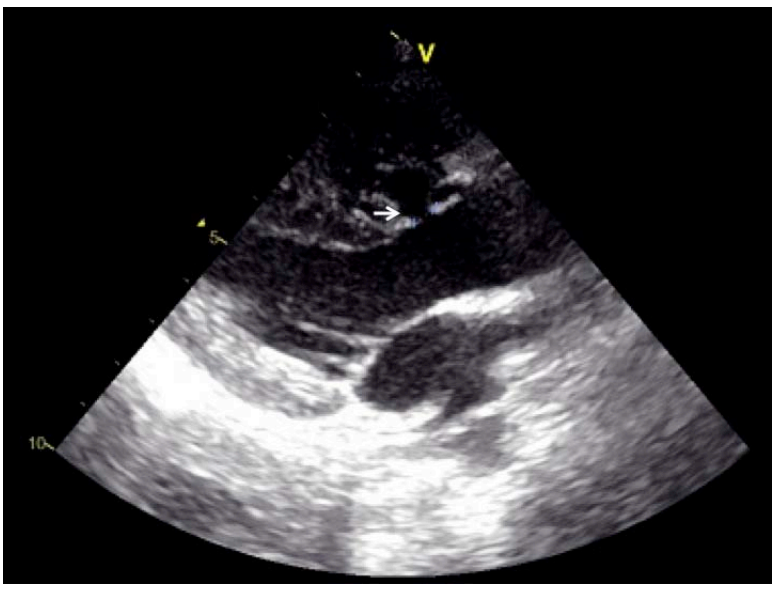

(a)

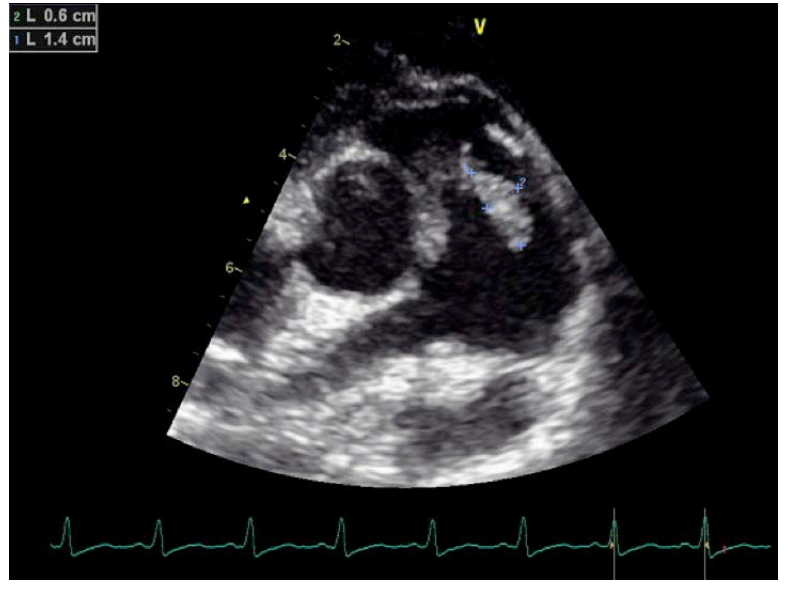

(b)

Figure 1. (a) Parasternal long axis view showing a perimembranous restrictive VSD and the aneurysm (arrow); (b) short axis view demonstrating the AMS obstructing the pulmonary trunk with small vegetation attached to its apex. Note the right ventricular hypertrophy and the absence of thrombus formation in the aneurysmal sac. 


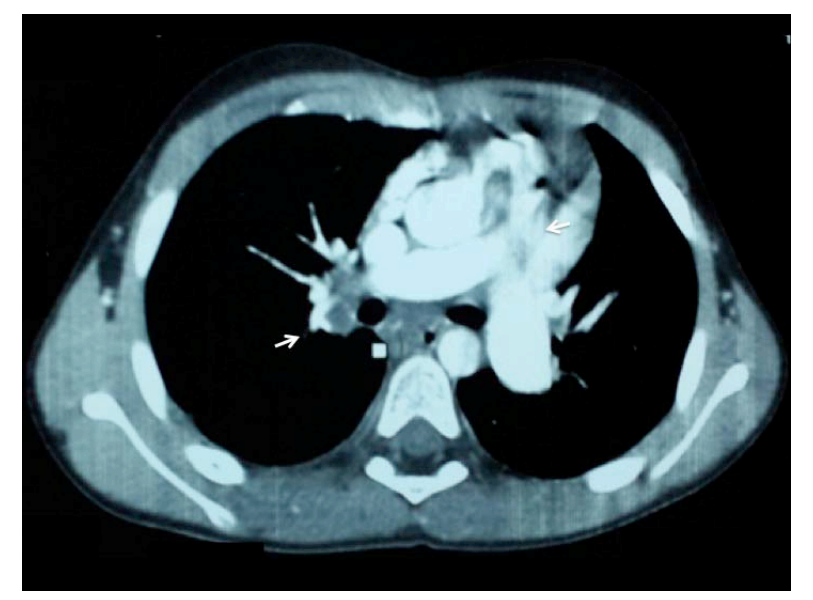

(a)

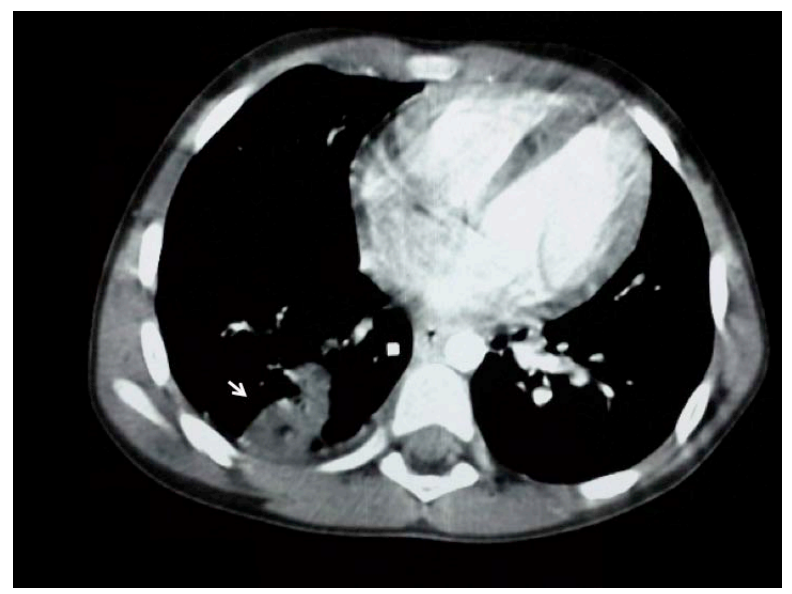

(b)

Figure 2. (a) Cardiac tomography shows the AMS bulging into the pulmonary trunk, the emboli obstructing the right inferior lobar artery (arrow); (b) pulmonary infarction (arrow).

globally infected aneurysmal sac without thrombus. There were some granulations in its apex suggestive of the presence of vegetations that were detached and migrated along the pulmonary artery tree causing, then, pulmonary infarction as seen in computed tomography angiography.

Unlike other reports where a thrombus had caused cerebral embolism [4], this is in our knowledge, the first described and documented case of a septic pulmonary embolism in the setting of AMS.

Although, patients with AMS have historically been considered not to require surgery, more recent data suggest that a significant percentage of these patients develop serious complications during adulthood [2] and thus require high risky surgery.

Because of the natural history of the disease and the lack of guidelines concerning management of this rare condition, surgical closure for small VSD with or without aneurysm may be a reasonable option.

\section{References}

[1] Anderson, R.H., Lenox, C.C. and Zuberbuhler, J.R. (1983) Mechanisms of Closure of Perimembranous Ventricular Septal Defect. The American Journal of Cardiology, 52, 341-345. http://dx.doi.org/10.1016/0002-9149(83)90135-2

[2] Yilmaz, A.T., Ozal, E., Arslan, M., Tatar, H. and Ozturk, O.Y. (1997) Aneurysm of the Membranous Septum in Adult Patients with Perimembranous Ventricular Septal Defect. European Journal Cardio-Thoracic Surgery, 11, 307-311. http://dx.doi.org/10.1016/S1010-7940(96)01058-5

[3] Afaneh, A.B., Wymer, D.C., Kraft, S. and Winchester, D.E. (2012) Membranous Ventricular Septal Aneurysm Diagnosed by Means of Cardiac Computed Tomography. Texas Heart Institute Journal, 39, 450-451.

[4] Salazar, J., Gutierrez, A., Cay, E., Ballester, C., Salazar, J.J. and Placer, L. (2003) Cerebral Embolism and Thrombus in a Membranous Interventricular Septal Aneurysm. The Annals of Thoracic Surgery, 76, 286-287. http://dx.doi.org/10.1016/S0003-4975(03)00152-8 